\title{
Structure and polymerization dynamics of bacterial actin MreB3 and MreB5 involved in Spiroplasma swimming.
}

\author{
Daichi Takahashi ${ }^{1}$, Ikuko Fujiwara ${ }^{1,2,3, *}$, Yuya Sasajima ${ }^{1}$, Akihiro Narita $^{4}$, Katsumi Imada ${ }^{5}$, Makoto \\ Miyata ${ }^{1,2, *}$ \\ 1. Graduate School of Science, Osaka City University, Osaka, Japan. \\ 2. The OCU Advanced Research Institute for Natural Science and Technology (OCARINA), \\ Osaka City University, Osaka, Japan \\ 3. Department of Bioengineering, Nagaoka University of Technology, Nagaoka, Niigata, Japan \\ 4. Graduate School of Science, Nagoya University, Nagoya, Japan \\ 5. Graduate School of Science, Osaka University, Osaka, Japan \\ * Ikuko Fujiwara, Makoto Miyata \\ Email: ikukofujiwara@vos.nagaokaut.ac.jp miyata@osaka-cu.ac.jp
}

Author Contributions: Experimental design was performed by DT, IF, and MM. The data correction of X-ray diffraction experiments and structural determination were performed by KI. EM image analyses were performed by DT, AN, and YS. All other experiments and data analyses were performed by DT. The manuscript was written by DT, IF, and MM, and then completed by all authors.

Competing Interest Statement: The authors declare no conflict of interest.

Classification: Major category: Biological Sciences, Minor category: Biophysics and Computational Biology

Keywords: bacterial cytoskeleton, ATPase, X-ray crystallography, electron microscopy, Mollicutes

\begin{abstract}
MreB is a bacterial protein belonging to the actin superfamily. It polymerizes into an antiparallel double-stranded filament that generally functions for cell shape determinations by maintaining the cell wall synthesis. Spiroplasma eriocheiris, a helical wall-less bacterium, has five classes of MreB homologs (SpeMreB1-5) that are responsible for its swimming motility. SpeMreB5 is likely responsible for generating the driving force for the swimming motility. However, molecular profiles involved in the swimming motility are poorly understood. Additionally, SpeMreB3 has distinct sequence features from the other SpeMreBs. Here, we have revealed the structures and polymerization dynamics of SpeMreB3 and SpeMreB5. Both SpeMreBs formed antiparallel double-stranded filaments with different characters; SpeMreB3 formed short filaments with slow polymerization, and SpeMreB5 filaments further assembled into bundle structures such as raft and paracrystal. SpeMreB5 filaments hydrolyzed ATP at a constant rate and were depolymerized immediately after ATP depletion. The Pi release rate of SpeMreB3 was much slower than that of SpeMreB5. Our crystal structure of SpeMreB3 and $P_{i}$ release measurements of SpeMreB3 and SpeMreB5 mutant variants explain that the cause of the slow $P_{i}$ release is the lack of the amino acid motif "E $\cdots T$ - X - [DE]", found in almost all MreBs, which probably takes roles to adjust the position and eliminate a proton of the putative nucleophilic water for $y-P_{i}$ of AMPPNP. These results show that SpeMreB3 has unique polymerization dynamics without bundle formations, whereas SpeMreB5 shows bundle formations, and its polymerization dynamics occur in the same manner as other actin superfamily members.
\end{abstract}




\section{Significance Statement}

MreB, a member of the actin superfamily, is widely conserved as a single copy on the genomes in bacteria having cell walls. It forms filaments and functions as a part of a protein complex for cell wall maintenance. While the number of genes and the cellular function of MreB are widely conserved for all bacterial phyla, several MreBs defying the conventional characteristics have been found. Spiroplasma, wall-less bacteria, have at least five MreBs (MreB1-5) in the genome and are thought to use the MreBs for the swimming motility of the cell. Here, we performed structural and biochemical analyses on two of five MreBs (MreB3 and MreB5), and found unique structural and polymerization features.

\section{Main Text}

\section{Introduction}

MreB, a protein in the actin superfamily, can be found in many walled bacteria characterized by an elongated morphology $(1,2)$. Previous studies reported that MreB forms an antiparallel double-stranded filament $(3,4)$. MreB possesses a nucleotide binding cleft, and binds ATP and GTP (5-7). Through the conformational change upon the polymerization, nucleotide hydrolysis is facilitated by the reassignment of the positions of the conserved glutamate and threonine interacting with the putative nucleophilic water for $y-P_{i}(4,8)$. Although the residues for nucleotide hydrolysis are found in many MreBs $(4,9)$, more detailed insights for the nucleotide hydrolysis mechanism and the role of nucleotide hydrolysis for the polymerization dynamics are required. In bacterial cells, MreB filaments bind to the cell membrane directly and function as a scaffold of an "elongasome" complex for synthesis of peptidoglycan, bacterial cell-wall $(2,10)$. MreB filaments move in a direction perpendicular to the cell axis, and the movement is coupled with the peptidoglycan synthesis (11). However, some MreBs take distinct roles besides cell wall synthesis, such as Myxococcus xanthus MreB driving cell gliding (12) and Helicobacter pylori MreB involved in chromosome segregation and urease activity (13). Recently, new members of MreB proteins involved in swimming motility have been found in the Spiroplasma species $(9,14)$. Spiroplasma belong to the class Mollicutes which evolved from the phylum Firmicutes, which also includes Bacillus subtilis (15-17). They have a helical-shaped cell lacking the peptidoglycan layer, and show a unique swimming motility unrelated to major bacterial motilities such as flagella, which generate propulsive force through a uni-directional rotation: the switching of the cell helicity is generated at the cell tip and transmitted along the cell axis to rotate the cell body $(15,18-21)$. The helicity switching and its transmission are probably caused by conformational changes of the internal helical ribbon structure extending along the entire cell axis $(18,22-24)$. The ribbon structure is thought to be composed of fibril, a cytoskeletal protein unique to Spiroplasma $(18,22$, 23), and five classes of MreB proteins (MreB1-5) $(24,25)$. A recent study determined the crystal structure of Spiroplasma citri MreB5 (SciMreB5), and revealed that SciMreB5 is essential for helicity formation and swimming motility (3).

The five classes of Spiroplasma MreBs are phylogenetically distinct from the walled-bacterial MreBs and featured differently $(9,14)$. Among them, MreB3 has distinct sequence character. In particular, conserved amino acid residues for ATP hydrolysis are substituted from glutamate and threonine to aspartate and lysine, respectively (Fig. S1A), which can be expected to show a slow ATPase activity and play a unique role in the Spiroplasma swimming $(9,14)$. However, molecular profiles of the MreBs involved in the swimming motility are poorly understood. Here, we studied the structure and polymerization dynamics of MreB3 and MreB5 of Spiroplasma eriocheiris (SpeMreB3 and SpeMreB5), a model Spiroplasma for studies of the swimming motility $(18,19$, $22,26,27)$. SpeMreB3 formed filaments without bundle formations and showed low ATPase activity, whereas SpeMreB5 formed bundle structures and showed polymerization characters common in the actin superfamily.

\section{Results}

\section{Filament structures of SpeMreB3 and SpeMreB5}

SpeMreB3 and SpeMreB5 were individually expressed in Escherichia coli and purified as 
monomers (Fig. S2). To examine the polymerization ability, they were incubated in standard buffer (20 mM Tris- $\mathrm{HCl} \mathrm{pH} \mathrm{7.5,} 100 \mathrm{mM} \mathrm{KCl,} 5 \mathrm{mM}$ DTT, and $2 \mathrm{mM} \mathrm{Mg-ATP)}$ for 3 hours at 24$27^{\circ} \mathrm{C}$ and observed by negative staining electron microscopy (EM). The EM images showed that SpeMreB3 formed short double-stranded filaments, in which $90 \%$ were $30-80 \mathrm{~nm}$ long and the median of length was $56.3 \mathrm{~nm}$ (Figs. 1A, S3A). The filaments were also observed in the presence of $2 \mathrm{mM} \mathrm{Mg-ADP}$ (Fig. S3B). In contrast, only small numbers of filaments were observed in nucleotide free (Nf) conditions (Fig. S3C\&D). These results indicate that SpeMreB3 polymerization depends on nucleotide states. To analyze the filament structure, around 2000 filament images were picked by the boxer program in EMAN ver. $2.2(28,29)$ and averaged by RELION ver. 3.0 (30). The averaged image showed that SpeMreB3 forms antiparallel doublestranded filaments with a subunit repeat of $5.3 \pm 0.2 \mathrm{~nm}$ (Figs. 1B, S3E-G). The protofilaments in the double-stranded filaments are bridged by weak density. While the antiparallel polarity and the subunit repeat are similar to those of the MreB filament from Caulobacter crescentus (CcMreB), a walled-bacterium (4), the filament maximum width was $8.7 \pm 0.1 \mathrm{~nm}$, which is about $2 \mathrm{~nm}$ wider than that of CcMreB.

In contrast, SpeMreB5 filaments formed bundles with two distinct structures. In the presence of 2 $\mathrm{mM}$ Mg-ATP, SpeMreB5 polymerized to raft structures at the protein concentration of $5-7.5 \mu \mathrm{M}$ and to paracrystalline structures at the protein concentration of $10 \mu \mathrm{M}$ (Figs. 1C\&D, S3I). The raft structures were also found in the presence of $2 \mathrm{mM} \mathrm{Mg-ADP}$ (Fig. S3J). However, no rafts nor filaments were observed under Nf-conditions (Fig. S3K\&L). The paracrystals were not observed in the absence of $\mathrm{Mg}^{2+}$ (Fig. S3M), whereas $0.5 \mathrm{mM} \mathrm{Mg}^{2+}$ was sufficient to form the paracrystals (Fig. S3N). The averaged image from around 1400 raft images showed that the raft is $17.3 \pm 0.1$ $\mathrm{nm}$ wide and composed of four protofilaments with a subunit repeat of $5.3 \pm 0.1 \mathrm{~nm}$ along the filament (Fig. 1E). Some image classes showed rafts consisting of three protofilaments, in which the width is $13.8 \pm 0.2 \mathrm{~nm}$, and two out of three protofilaments can be fit by the central two protofilaments in the four-stranded raft (Fig. S3O\&P), suggesting that the outer protofilaments are less stable than the central pair in the four-stranded raft. The central two protofilaments showed antiparallel polarity and can be fit by the structure of CcMreB double strands ( $\sim 6.5 \mathrm{~nm})$ (Fig. S3Q\&R) $(4,10)$, suggesting that the structure of the central protofilament pair is equivalent to the CcMreB filament. Our results indicate that the raft consists of the antiparallel double-stranded filament and peripheral protofilaments.

\section{Crystal structures of SpeMreB3}

SpeMreB3 crystals suitable for X-ray experiments grew in several conditions but showed merohedral twining. To overcome the twining problem, we methylated lysine residues of SpeMreB3 and crystallized it (Fig. S4A\&B) (31, 32). The crystal structures of Nf-SpeMreB3 and its AMPPNP complex were determined at 1.90 and $1.75 \AA$ resolution, respectively (Fig. $1 \mathrm{~F}$, Table S1). SpeMreB3 adopts the canonical actin fold composed of four subdomains (IA, IB, IIA, and IIB) (33) and consists of the same secondary structure elements as in CcMreB except for the very Cterminal region (4).

The Nf-SpeMreB3 crystal belongs to a space group of $P 2{ }_{1}$ and contains a single molecule in an asymmetric unit. Nf-SpeMreB3 forms filaments along the crystal a axis in the $P 2{ }_{1}$ crystal, and thus the filaments are arranged in an antiparallel manner. The subunit arrangement in the filament resembles those in the CcMreB and SciMreB5 filaments in their respective crystals (Fig. 1F) $(3,4)$. However, the interaction between IIA and IB' (with and without a prime indicate $i$ and $i-$ 1 subunits, respectively), which is observed in the CcMreB and SciMreB5 filaments, does not exist in the Nf-SpeMreB3 filament, whereas the IIA-IIB' interaction is conserved in Nf-SpeMreB3 (Fig. 1G). The IIA-IIB' interaction is mediated by a hydrogen bonding network and stabilized by an electrostatic interaction between E285 and the N-terminal end of the a-helix starting from V216'. The SpeMreB3 AMPPNP complex crystal includes two molecules (Mol-A and B) (Fig. 1F), which are related by pseudo-two-fold symmetry axis perpendicular to the crystal a axis, in an asymmetric unit. Each SpeMreB3 molecule in the asymmetric units forms a filament with those in the neighboring unit cells along the crystal a axis. Thus, the SpeMreB3 AMPPNP complex crystal also contains antiparallel filaments, but their arrangement differs from that in the Nf-SpeMreB3 
crystal. The two molecules show small difference in domain conformation in which the nucleotide binding cleft in Mol-B is slightly wider than that in Mol-A (Fig. 1I). Compared with Nf-SpeMreB3, both clefts are narrower, and the overall conformation is more flattened (Fig. 1I). The IIA-IIB' interaction area is wider than that of Nf-SpeMreB3 (Fig. 1G\&H). As observed in Nf-SpeMreB3, E285 in the SpeMreB3 AMPPNP complex interacts electrostatically with the N-terminal end of the a-helix starting from V216'. Like CcMreB and SciMreB5 filaments $(3,4)$, IIA in the SpeMreB3 AMPPNP complex also interacts with IB', in which the interaction is mediated through a hydrogen bonding network and stabilized by an electrostatic interaction between D289 and K70'. The subunit repeat along the filament in the crystal is in good agreement with that of the protofilament in the EM image (Fig. 1B\&F). Therefore, we fit the filament model in the crystal onto the 2D averaged EM image of the SpeMreB3 filament. A single filament model of SpeMreB3 AMPPNP complex was fit well to the protofilament image (Figs. $1 \mathrm{~J}, \mathrm{~S} 3 \mathrm{H}$ ), suggesting that the protofilament in the double-stranded filament (Fig. 1B) takes similar structure to that in the crystal. However, any antiparallel filament pairs in the crystal did not fit to the double-stranded filament EM image, indicating that the interaction stabilizing the double-stranded filament in solution is different from that in the crystal.

The surface charge distribution of the SpeMreB3 protofilament greatly differs from that of SciMreB5 (Fig. 1K). The IA and IIA subdomains of the SciMreB5 protofilament have highly negatively charged surfaces. This is probably true for SpeMreB5 because the amino acid sequences of SciMreB5 and SpeMreB5 are $87.5 \%$ identical and residues in the protofilament surface are highly conserved (Fig. S1B-D). However, the corresponding surfaces of the SpeMreB3 protofilament show both positive and negative charge. These features on surface charge may cause the difference in the bundle formation pattern between filaments of SpeMreB3 and SpeMreB5 (Fig. 1A-E).

\section{SpeMreB3 and SpeMreB5 show different polymerization modes}

Next, we examined the polymerization dynamics of SpeMreB3 and SpeMreB5 at various concentrations (1-12 $\mu \mathrm{M})$ using light scattering at $90^{\circ}$ angle (Fig. 2A\&B). The polymerization was initiated by adding $2 \mathrm{mM} \mathrm{Mg}$-ATP at $25^{\circ} \mathrm{C}$. At $1 \mu \mathrm{M}$, lag phases were observed in both SpeMreBs, indicating that the assembly dynamics of the SpeMreBs proceed in two stages, nucleation and polymerization phases, like other cytoskeletal proteins (34). To observe the plateau, we continued light scattering experiments for 2 hours (Fig. 2C). SpeMreB3 reached a plateau around 90 min at $10 \mu \mathrm{M}$. Polymerization of SpeMreB5 was biphasic at $10 \mu \mathrm{M}$ whereas it was monophasic at $5 \mu \mathrm{M}$. EM observation of $10 \mu \mathrm{M}$ SpeMreB5 at the first phase showed only raft structures (Fig. S3S), unlike $10 \mu \mathrm{M}$ SpeMreB5 at the steady-state in which paracrystals were observed (Fig. 1D). These results indicate that the second phase corresponds to the formation of the paracrystals of SpeMreB5.

\section{Critical concentration for polymerization estimated by light scattering and sedimentation assays} We evaluated the critical concentrations of SpeMreB3 and SpeMreB5 (Fig. 2D). From the $x$ intercept of linear fitting of the light scattering intensities at steady state over each SpeMreB concentration, critical concentrations of SpeMreB3 and SpeMreB5 were estimated as $0.66 \pm 0.28$ and $0.42 \pm 0.05 \mu \mathrm{M}$, respectively. These values are similar to those of walled-bacterial MreBs (58).

We also determined the critical concentrations by sedimentation assay. The SpeMreBs were polymerized in the standard buffer at $24-27^{\circ} \mathrm{C}$ and subjected to ultracentrifugation $(264,000 \times g$, $120 \mathrm{~min}$ ) to separate monomers and filaments (Fig. 3A\&B). Both SpeMreBs showed critical concentrations similar to those estimated from the light scattering assay (Fig. 3C). While more than $80 \%$ of the total amount of SpeMreB5 appeared in the pellet fractions (Fig. 3B\&C), the ratios of pelleted SpeMreB3 were less than 58\% (Fig. 3A\&C). To understand this difference, we examined the concentration gradation in the supernatant (Fig. 3D). SpeMreB3 at $10 \mu \mathrm{M}$ concentration was polymerized for 3 hours and subjected to ultracentrifugation. Then, the supernatant was divided into upper and bottom halves as shown in figure 3D. The concentration 
of the upper half was $0.79 \pm 0.39 \mu \mathrm{M}$, similar to the critical concentration. However, the concentration of the bottom half was $8.14 \pm 1.14 \mu \mathrm{M}, 10.3$ times higher than that of the upper half. These results indicate that SpeMreB3 forms short filaments. In addition, the concentration of the whole supernatant $(4.47 \pm 0.72 \mu \mathrm{M})$, a mixture of both top and bottom, is not significantly different to that of the pellet resuspended in a buffer equivalent amount to the sample $(5.53 \pm$ $0.72 \mu \mathrm{M}$ ), suggesting that half of SpeMreB3 filaments were not large enough to be pelleted.

\section{Different $P_{i}$ release rates of SpeMreBs resulting from structural differences in active site for ATP hydrolysis}

Previous studies showed that the polymerization dynamics of MreBs are coupled with ATP hydrolysis $(4,8)$. Thus, we measured ATPase activities of SpeMreB3 and SpeMreB5 by the $P_{i}$ release assay during polymerization. The measurement was initiated by adding Mg-ATP to $3 \mu \mathrm{M}$ SpeMreBs in the standard buffer, and released $\mathrm{P}_{\mathrm{i}}$ was traced by the absorbance of 2-amino-6mercapto-7-methylpurine riboside (MESG), a molecular probe for $\mathrm{P}_{\mathrm{i}}(8,35,36)$. In the presence of $2 \mathrm{mM} \mathrm{Mg-ATP}$, SpeMreB5 released $\mathrm{P}_{\mathrm{i}}$ in a constant rate over time even after the light scattering intensity plateaued (Fig. 4A). The Pidissociation rate constant of SpeMreB5 was $1.5 \pm$ $0.2 \mathrm{nM}\left(\mathrm{P}_{\mathrm{i}}\right) / \mathrm{s} / \mu \mathrm{M}$ (protein) (Figs. 4B, S5A). In the ATP concentration equivalent to the protein concentration $(3 \mu \mathrm{M})$, the $P_{i}$ release of SpeMreB5 proceeded at a constant rate and then stopped due to ATP depletion. After this stall of $P_{i}$ release, the light scattering intensity reflecting the amount of polymerized SpeMreB5 decreased (Fig. 4C). The critical concentration after ATP depletion, namely SpeMreB5 in ADP form, was 13 times higher than that under 2 mM ATP (Fig. 4D\&E).

As expected from the sequence analyses (9), the $P_{i}$ release of SpeMreB3 was 33 times slower than that of SpeMreB5 (Fig. 4A\&B). To elucidate the structural basis for the slow $\mathrm{P}_{\mathrm{i}}$ release rate, we compared the AMPPNP complex structure of SpeMreB3 with those of CcMreB (PDB: 4CZJ) and SciMreB5 (PDB: 7BVY) (Fig. 4F-H). In the active site of CcMreB, E140 and T167 coordinate a water molecule, which is believed to nucleophilically attack $\mathrm{Y}-\mathrm{P}_{\mathrm{i}}$ of ATP, and T167 interacts with E169 (Fig. 4F) (4). These residues are structurally conserved in SciMreB5 (E134, T161, and $\mathrm{D} 163$ in Fig. 4H), although no water molecule corresponding to the nucleophilic water was observed. In SpeMreB3, D147, corresponding to E140 in CcMreB, is distant from the putative nucleophilic water molecule compared with that of $\mathrm{CcMreB}$, and K174, corresponding to T167 in CcMreB, does not interact with the water molecule (Fig. 4G). Moreover, the residue corresponding to E169 in CcMreB is replaced by serine (S176) in SpeMreB3 (Fig. 4G). Therefore, these three residues may attribute to the slow $P_{i}$ release rate of SpeMreB3.

To elucidate the role of these residues on ATP hydrolysis, we prepared four mutant variants of SpeMreB3 (SpeMreB3 ${ }^{\mathrm{D} 147 \mathrm{E}}$, SpeMreB3 ${ }^{\mathrm{K} 174 T}$, SpeMreB3 ${ }^{\text {S176D }}$, and SpeMreB3 ${ }^{\mathrm{K} 174 \mathrm{~T} / \mathrm{S} 176 \mathrm{D})}$ ) and measured their $P_{i}$ release rates. These mutant variants formed double-stranded filaments (Fig. S3T-W), indicating that the mutations do not affect polymerization ability. The D147E mutation increased the $P_{i}$ release rate three times higher than that of wild type, whereas the K174T and S176D mutations did not (Figs. 4A\&B, S5B). In contrast, the K174T/S176D double mutation increased the $P_{i}$ release rate of SpeMreB3 11 times higher than that of wild type (Figs. 4A\&B, S5B). These results indicate that the Thr - Asp pair is important for ATP hydrolysis and plays a distinct role from the glutamate at the position corresponding to E140 in CcMreB. The importance of the Thr - Asp pair on ATP hydrolysis is further confirmed by mutation analysis of SpeMreB5 using mutant variants of SpeMreB5 ${ }^{\mathrm{T} 160 \mathrm{~A}}$ and SpeMreB5 ${ }^{\mathrm{D} 162 \mathrm{~S}}$, where T160 and D162 are corresponding to T161 and D163 in SciMreB5 (Fig. 4H). The $P_{i}$ release rate of SpeMreB5D162S was $18 \%$ of that of wild type, and that of SpeMreB5 ${ }^{\mathrm{T} 160 \mathrm{~A}}$ was only $2 \%$ (Figs. $4 \mathrm{~A} \& \mathrm{~B}, \mathrm{~S} 5 \mathrm{C}$ ), while the polymerization abilities were not affected by the mutations (Fig. S3X\&Y).

Our $\mathrm{P}_{\mathrm{i}}$ release measurements coordinated with analyses of crystal structures (Figs. 4A-H, S5) showed that the amino acid motif "E $\cdots T$ - X - [DE]" plays a crucial role for ATP hydrolysis of MreB. This motif is conserved in $95.8 \%$ of MreBs of non-Spiroplasma species including all bacterial phyla and in $98.2 \%$ of Spiroplasma MreBs except for MreB3 (Fig. 4l left and right). In all Spiroplasma MreB3, the residues corresponding to E140 and T167 in CcMreB are replaced by 
aspartate and lysine, respectively, and the residue corresponding to E169 in CcMreB is replaced by serine or threonine (Fig. 4 I center) (9).

\section{Discussion \\ Characters of SpeMreB3 and SpeMreB5 filaments}

The present study revealed that SpeMreB3 and SpeMreB5 have different filament structures and polymerization dynamics. SpeMreB3 formed an antiparallel double-stranded filament with the subunit repeat similar to that of the CcMreB filament (4). However, the width of the SpeMreB3 filament $(8.7 \mathrm{~nm})$ was about $2 \mathrm{~nm}$ wider than that of the CcMreB filament $(6.5 \mathrm{~nm})$ (Fig. 1B) (4). Although the protofilament structure in the crystal was fit well in the EM image of the doublestranded filament, only L125 is involved in the direct inter-protofilament interaction in the atomic model of the double-stranded filament (Figs. 1J, S3H). The typical contact area for interprotofilament interaction of other actin superfamily proteins is $700-900 \AA^{2}(37)$. Therefore, the protofilament structure in the crystal probably slightly differs from that in the double-stranded filament. The lengths of SpeMreB3 filaments were distributed around $56.3 \mathrm{~nm}$ (Fig. S3A), much shorter than walled-bacterial MreBs forming filaments with lengths of micro-meter order in vitro (5, $6,8)$. In addition, the sedimentation assays showed that $58 \%$ of SpeMreB3 remained in the supernatant (Fig. 3A\&C\&D). These results suggest that SpeMreB3 cannot form long filaments. Such short filaments have been reported in the actin of Plasmodium, a human pathogenic protozoan, which is involved in the gliding motility of the cells as a track for the myosin motors (38-40).

SpeMreB5 filaments formed bundle structures such as rafts and paracrystals (Figs. 1C-E, S3I\&M\&N). Raft structures have been observed in walled-bacterial MreBs $(6,41)$, although their roles in the cell remain unclear. In Spiroplasma cells, it was observed that there are two types of filaments both of which laterally align to form a sheet structure in 4 and $11 \mathrm{~nm}$ intervals. Thicker filament is thought to be composed of fibril based on dimensions of isolated fibril filaments (24, 25). Thinner filament is thought to consist of MreBs based on recent proteome analyses $(18,22)$, however it has not been confirmed yet. Our study showed that both widths of three-stranded and four-stranded rafts of SpeMreB5 (13.8 \pm 0.2 and $17.3 \pm 0.1 \mathrm{~nm}$, respectively) are consistent with that of intra-cellular thinner filaments $(13.8 \mathrm{~nm} \div 3=4.5 \mathrm{~nm}$ and $17.3 \mathrm{~nm} \div 4=4.3 \mathrm{~nm}$, respectively), suggesting that the SpeMreB5 rafts may be associated laterally aligning thinner filaments in the cells. The paracrystalline formation of SpeMreB5 is $\mathrm{Mg}^{2+}$ dependent (Fig. S3M\&N), suggesting that the SpeMreB5 filaments are bridged by $\mathrm{Mg}^{2+}$ to form the paracrystals, like skeletal actin filaments $(42,43)$. $\mathrm{Mg}^{2+}$ probably binds to subdomains IA or IIA of SpeMreB5 because these subdomains have a broad negatively charged surface (Figs. 1K lower, S1D). SpeMreB3 has no such negatively charged surface (Fig. 1K upper), consistent with the no paracrystal formations (Fig. 1A). In skeletal actin filaments, divalent cations for bundle formations are suggested to bind to six acidic residues located on the sides of subdomains IA and IB (42). However, these residues are not conserved in SpeMreB5. Moreover, the charge distribution of the corresponding surface of SpeMreB5 is different from that of skeletal actin, suggesting that the paracrystal formation of SpeMreB5 filaments is mediated by a different interaction pattern to that of skeletal actin filaments.

\section{ATP hydrolysis mechanism of MreB family proteins}

A previous study showed that E140 and T167 in CcMreB align the nucleophilic water to an appropriate position for ATP hydrolysis (4), although it was not clear which residue takes a role in proton elimination from the water molecule, which is a necessary step for ATP hydrolysis. Through our Pirelease assays of SpeMreB3 and SpeMreB5 (Figs. 4A\&B, S5) and comparisons of crystal structures and sequences of MreBs (Fig. 4F-I), we showed that the threonine - [either of aspartate or glutamate] residue pair and the conserved glutamate corresponding to E140 in CcMreB play distinct roles for ATP hydrolysis. On the basis of these findings, we update the role of active site residues on ATP hydrolysis using CcMreB as a model. E169 eliminates the proton 
of the side chain hydroxy group of T167 to activate it as an acidic catalyst for proton elimination of the nucleophilic water. E140 adjusts the position of the nucleophilic water suitable for attacking the $\mathrm{Y}^{-} \mathrm{P}_{\mathrm{i}}$ of ATP (Fig. 4F). This reaction mechanism is akin to that proposed for skeletal actin, where Q137, corresponding to E140 in CcMreB, is responsible for the positioning of the nucleophilic water, and $\mathrm{H} 161$, corresponding to T167 in CcMreB, is responsible for the proton elimination of the nucleophilic water (44). The core amino acid motif " $E \cdots T$ - X - [DE]" is mostly conserved in MreBs except for Spiroplasma MreB3 (Fig. 4l left and right), suggesting that the ATP hydrolysis mechanism proposed here is conserved in most of MreBs except for Spiroplasma MreB3.

\section{Effect of SpeMreB3 methylation on the polymerization}

We methylated lysine residues to obtain the SpeMreB3 crystals suitable for the structural determination (Fig. S4A\&B). Methylated SpeMreB3 formed double-stranded filaments as observed in unmethylated filaments (Fig. S4C). Nevertheless, the critical concentration of methylated SpeMreB3 was about 60-fold higher than that of the unmethylated ones (Fig. S4D\&E). One of the causes may be due to the inhibitions of lysine residue-mediated interactions involved in the filament formation. Our crystal structure of the SpeMreB3 AMPPNP complex showed that K70, involved in the intra-protofilament interaction, is di-methylated (Fig. $1 \mathrm{H})$. In methylated SpeMreB3, this interaction may be disturbed in solution conditions probably due to the decrease of degree of freedom for the interaction. In the structure of SpeMreB3 AMPPNP complex, $\mathrm{K} 174$ is di-methylated, and the methylation might change the position of the residue preventing interaction with the nucleophilic water (Fig. 4G). However, we do not think that this methylation greatly changes the ATPase activity of SpeMreB3, because the $P_{i}$ release rate was not changed by introducing the single K174T mutation (Figs. 4A\&B\&G, S5B).

Coupling of polymerization dynamics with ATPase activity in SpeMreB3 and SpeMreB5 SpeMreB3 forms short filaments with slow polymerization and $P_{i}$ release (Fig. 5A). SpeMreB3 polymerization was observed even in the absence of ATP (Fig. S3C\&D). This is consistent with the formation of protofilaments in the crystal of Nf-SpeMreB3 (Fig. 1G\&H). These results suggest that ATP is not the polymerization initiator of SpeMreB3 as observed in some walled-bacterial MreBs (7).

In contrast, SpeMreB5 polymerizes with ATP and hydrolyzes ATP much faster than SpeMreB3 (Fig. 5B). SpeMreB5 filaments depolymerized immediately after the stall of $P_{i}$ release (Fig. 4C), indicating that SpeMreB5 exchanges the subunits by ATP hydrolysis. Subunit recycling is found in many actin superfamily proteins (45-48), although it has not yet been clarified for MreBs. In addition, the critical concentration of ADP-SpeMreB5 was 13 times higher than that of ATPSpeMreB5 (Fig. 4D\&E). This difference is similar to skeletal actin, whose critical concentration in the ADP bound state is 18 times higher than that in the ATP bound state $(46,48)$. These results indicate that subunit dissociation in the SpeMreB5 filaments is triggered by $\mathrm{Pi}_{\mathrm{i}}$ release.

\section{Roles of SpeMreB3 and SpeMreB5 for Spiroplasma swimming}

Our current work revealed that SpeMreB3 has unique characters among actin superfamily proteins. What is the cellular function of it? Previous studies on walled-bacterial MreBs suggested that in vitro conditions are more suitable for the polymerizations than intracellular conditions $(7$, 8). Considering that SpeMreB3 filaments are short in in vitro conditions (Figs. 3A\&C\&D, S3A), SpeMreB3 may form oligomeric filaments or take monomeric states in cells. The impairment of the core amino acid motif for ATP hydrolysis (Figs. 4F-I, S1A) suggests that the subunits in the SpeMreB3 filaments are rarely exchanged. Taken together with the presence of an amphipathic helix at the N-terminus (Fig. S1A) (9), SpeMreB3 may assemble the ribbon structure to the cell membrane statically. In fact, MreB3 is found in the membrane fraction of $S$. citri (3).

Spiroplasma have been thought to swim by changing the conformation of the intracellular ribbon structure composed of fibril and several types of MreBs $(18,22-24)$. What is the direct energy source for the conformational changes? Either membrane potential or ATP is normally utilized as the direct energy source for driving bacterial motility machinery (15). For Spiroplasma swimming, 
ATP is likely the energy source, because the membrane potential of Spiroplasma is $-68 \mathrm{mV}$, much less than that of walled bacteria having flagella motors $(-150 \mathrm{mV})$, which are driven by proton motive force (49-51). In fact, the gliding motility of two Mycoplasma species, belonging to the class Mollicutes as well as Spiroplasma, are driven by ATP $(52,53)$. Fibril, one of the main components of the ribbon, does not have ATPase activity (18). In contrast, SpeMreB5 has ATPase activity, and the activity is coupled with its polymerization dynamics. A recent study reported that SciMreB5 is essential for the swimming of the cell (3). Therefore, the polymerization dynamics of SpeMreB5 (Figs. 2B\&C, 4A-E) is probably related to the swimming motility of Spiroplasma directly.

\section{Materials and Methods}

The whole experimental procedures are described in SI Appendix. We describe them briefly here. The SpeMreBs were expressed as the fusion of the histidine-tag at the N-termini in E. coli str. BL21 (DE3) or C43 (DE3) by using pET or pCold expression systems, and purified by $\mathrm{Ni}^{2+}-\mathrm{NTA}$ affinity and gel filtration chromatography. The polymerization of SpeMreBs was performed with standard buffer $\left(20 \mathrm{mM}\right.$ Tris- $\mathrm{HCl} \mathrm{pH} 7.5$ at $25^{\circ} \mathrm{C}, 100 \mathrm{mM} \mathrm{KCl}, 5 \mathrm{mM}$ DTT, $2 \mathrm{mM} \mathrm{MgCl}$, and 2 $\mathrm{mM} \mathrm{Na} \mathrm{N}_{2}$ ATP) and buffers in which a concentration of a component was modified from the standard buffer. The steady state of SpeMreBs was created after 3 hours of polymerization at 24$27^{\circ} \mathrm{C}$. Sedimentation, light scattering, and $P_{i}$ release assays were performed with TLA-100 rotor (Beckman Coulter), FP-6200 (JASCO, Tokyo, Japan), and EnzChek kit (Thermo Fisher Scientific), respectively. For EM, the samples were stained with $2 \%(\mathrm{w} / \mathrm{v})$ uranyl acetate and observed under transmission electron microscope. The crystals of the nucleotide free (Nf)SpeMreB3 and SpeMreB3 AMPPNP complex were grown by sitting-drop vapor-diffusion technique, and measured at synchrotron beamlines in SPring-8 (Harima, Japan). The structural determinations were performed by molecular replacement by using structures of CcMreB (PDB: $4 \mathrm{CZL}$ ) and Nf-SpeMreB3 as the templates for Nf-SpeMreB3 and SpeMreB3 AMPPNP complex, respectively.

\section{Acknowledgments}

We thank Ms. Junko Shiomi and Ms. Tomomi Shimonaka for the technical assistance for SpeMreBs expression and MALDI-TOF MASS spectrometry, respectively, Dr. Yoshihiro Yamaguchi for providing E. coli str. C43 (DE3), Yuhei O Tahara, Takuma Toyonaga, and Masaki Mizutani for technical assistance with electron microscopy and its image analyses (all are affiliated to Graduate School of Science, Osaka City University), Dr. Norihiro Takekawa and Mr. Motoshi Sakai (Graduate School of Science, Osaka University) for technical assistance with the data collection of X-ray diffraction experiments, and Dr. Miki Kinoshita (Graduate School of Frontier Bioscience, Osaka University) for providing reagents for SpeMreB3 methylation. The English wording of the manuscript and SI appendix were confirmed by Dr. Timothy Day (Graduate School of Frontier Bioscience, Osaka University). This study was supported by a Grants-in-Aid for Scientific Research (A and C) (MEXT KAKENHI, Grant Numbers JP17H01544 to MM and JP20K06591 to IF), JST CREST (Grant Number JPMJCR19S5) to MM, Research Foundation of Opto-Science and Technology to IF, and the Osaka City University (OCU) Strategic Research Grant 2019 to IF. DT is a recipient of a Japanese scholarship of JEES Kureha (Toyobo) and Ono-Syougakukai Scholarships.

\section{References}

1. Yulo PRJ \& Hendrickson HL (2019) The evolution of spherical cell shape; progress and perspective. Biochem Soc Trans 47(6):1621-1634.

2. Shi H, Bratton BP, Gitai Z, \& Huang KC (2018) How to build a bacterial cell: MreB as the foreman of E. coli construction. Cell 172(6):1294-1305.

3. Harne S, et al. (2020) MreB5 is a determinant of rod-to-helical transition in the cell-wallless bacterium Spiroplasma. Curr Biol 30(23):4753-4762.e4757.

4. van den Ent F, Izoré T, Bharat TA, Johnson CM, \& Löwe J (2014) Bacterial actin MreB 
ms antiparallel double filaments. Elife 3:e02634

5. Nurse P \& Marians KJ (2013) Purification and characterization of Escherichia coli MreB protein. J Biol Chem 288(5):3469-3475.

6. Popp D, et al. (2010) Filament structure, organization, and dynamics in MreB sheets. $J$ Biol Chem 285(21):15858-15865.

7. Mayer JA \& Amann KJ (2009) Assembly properties of the Bacillus subtilis actin, MreB. Cell Motil Cytoskeleton 66(2):109-118.

8. Bean GJ \& Amann KJ (2008) Polymerization properties of the Thermotoga maritima actin MreB: roles of temperature, nucleotides, and ions. Biochemistry 47(2):826-835.

9. Takahashi D, Fujiwara I, \& Miyata M (2020) Phylogenetic origin and sequence features of MreB from the wall-less swimming bacteria Spiroplasma. Biochem Biophys Res Commun 533(4):638-644.

10. Salje J, van den Ent F, de Boer P, \& Löwe J (2011) Direct membrane binding by bacterial actin MreB. Mol Cell 43(3):478-487.

11. Billaudeau C, Yao ZZ, Cornilleau C, Carballido-López R, \& Chastanet A (2019) MreB forms subdiffraction nanofilaments during active growth in Bacillus subtilis. $m$ Bio 10(1):e01879-01818.

12. Fu G, et al. (2018) MotAB-like machinery drives the movement of MreB filaments during bacterial gliding motility. Proc Natl Acad Sci U S A 115(10):2484-2489.

13. Waidner B, et al. (2009) A novel system of cytoskeletal elements in the human pathogen Helicobacter pylori. PLoS Pathog 5(11):e1000669.

14. Ku C, Lo WS, \& Kuo CH (2014) Molecular evolution of the actin-like MreB protein gene family in wall-less bacteria. Biochem Biophys Res Commun 446(4):927-932.

15. Miyata M, et al. (2020) Tree of motility - A proposed history of motility systems in the tree of life. Genes Cells 25(1):6-21.

16. Castelle CJ \& Banfield JF (2018) Major new microbial groups expand diversity and alter our understanding of the tree of life. Cell 172(6):1181-1197.

17. Hug LA, et al. (2016) A new view of the tree of life. Nat Microbiol 1:16048.

18. Sasajima Y, Kato T, Miyata T, Namba K, \& Miyata M (2021) Elucidation of fibril structure responsible for swimming in Spiroplasma using electron microscopy. bioRxiv:2021.2002.2024.432793.

19. Nakane D, Ito T, \& Nishizaka T (2020) Coexistence of two chiral helices produces kink translation in Spiroplasma swimming. J Bacteriol 202(8):e00735-00719.

20. Wada H \& Netz RR (2009) Hydrodynamics of helical-shaped bacterial motility. Phys Rev E Stat Nonlin Soft Matter Phys 80(2 Pt 1):021921.

21. Shaevitz JW, Lee JY, \& Fletcher DA (2005) Spiroplasma swim by a processive change in body helicity. Cell 122(6):941-945.

22. Liu $\mathrm{P}$, et al. (2017) Chemotaxis without conventional two-component system, based on cell polarity and aerobic conditions in helicity-switching swimming of Spiroplasma eriocheiris. Front Microbiol 8:58.

23. Cohen-Krausz S, Cabahug PC, \& Trachtenberg S (2011) The monomeric, tetrameric, and fibrillar organization of Fib: the dynamic building block of the bacterial linear motor of Spiroplasma melliferum BC3. J Mol Biol 410(2):194-213.

24. Kürner J, Frangakis AS, \& Baumeister W (2005) Cryo-electron tomography reveals the cytoskeletal structure of Spiroplasma melliferum. Science 307(5708):436-438.

25. Trachtenberg S, et al. (2008) Structure of the cytoskeleton of Spiroplasma melliferum BC3 and its interactions with the cell membrane. J Mol Biol 378(4):778-789.

26. Terahara N, Tulum I, \& Miyata M (2017) Transformation of crustacean pathogenic bacterium Spiroplasma eriocheiris and expression of yellow fluorescent protein. Biochem Biophys Res Commun 487(3):488-493.

27. Wang W, Rong L, Gu W, Du K, \& Chen J (2003) Study on experimental infections of Spiroplasma from the Chinese mitten crab in crayfish, mice and embryonated chickens. Res Microbiol 154(10):677-680.

28. Bell JM, Chen M, Baldwin PR, \& Ludtke SJ (2016) High resolution single particle 
refinement in EMAN2.1. Methods 100:25-34.

29. Tang G, et al. (2007) EMAN2: an extensible image processing suite for electron microscopy. J Struct Biol 157(1):38-46.

30. Zivanov J, et al. (2018) New tools for automated high-resolution cryo-EM structure determination in RELION-3. Elife 7:7:e42166.

31. Shaw N, et al. (2007) (NZ)CH...O contacts assist crystallization of a ParB-like nuclease. BMC Struct Biol 7(7):46.

32. Walter TS, et al. (2006) Lysine methylation as a routine rescue strategy for protein crystallization. Structure 14(11):1617-1622.

33. Wagstaff J \& Löwe J (2018) Prokaryotic cytoskeletons: protein filaments organizing small cells. Nat Rev Microbiol 16(4):187-201.

34. Alberts B (2015) Molecular biology of the cell (Garland Science, Taylor and Francis Group, New York, NY) Sixth edition.

35. Blanchoin L \& Pollard TD (1999) Mechanism of interaction of Acanthamoeba actophorin (ADF/cofilin) with actin filaments. J Biol Chem 274(22):15538-15546.

36. Webb MR (1992) A continuous spectrophotometric assay for inorganic-phosphate and for measuring phosphate release kinetics in biological-systems. Proc Natl Acad Sci U S A 89(11):4884-4887.

37. Szewczak-Harris A \& Löwe J (2018) Cryo-EM reconstruction of AlfA from Bacillus subtilis reveals the structure of a simplified actin-like filament at 3.4-Å resolution. Proc Natl Acad Sci U S A 115(13):3458-3463.

38. Vahokoski J, et al. (2014) Structural differences explain diverse functions of Plasmodium actins. PLoS Pathog 10(4):e1004091.

39. Meissner M, Ferguson DJP, \& Frischknecht F (2013) Invasion factors of apicomplexan parasites: essential or redundant? Curr Opin Microbiol 16(4):438-444.

40. Schmitz S, et al. (2005) Malaria parasite actin filaments are very short. J Mol Biol 349(1):113-125.

41. van den Ent F, Amos LA, \& Löwe J (2001) Prokaryotic origin of the actin cytoskeleton. Nature 413(6851):39-44.

42. Castaneda N, et al. (2018) Cations modulate actin bundle mechanics, assembly dynamics, and structure. J Phys Chem B 122(14):3826-3835.

43. Popp D \& Robinson RC (2011) Many ways to build an actin filament. Molecular Microbiology 80(2):300-308.

44. Chou SZ \& Pollard TD (2019) Mechanism of actin polymerization revealed by cryo-EM structures of actin filaments with three different bound nucleotides. Proc Natl Acad Sci U $S$ A 116(10):4265-4274.

45. Ozyamak E, Kollman J, Agard DA, \& Komeili A (2013) The bacterial actin MamK: in vitro assembly behavior and filament architecture. J Biol Chem 288(6):4265-4277.

46. Fujiwara I, Vavylonis D, \& Pollard TD (2007) Polymerization kinetics of ADP- and ADP-Piactin determined by fluorescence microscopy. Proc Natl Acad Sci U S A 104(21):88278832.

47. Garner EC, Campbell CS, \& Mullins RD (2004) Dynamic instability in a DNA-segregating prokaryotic actin homolog. Science 306(5698):1021-1025.

48. Pollard TD (1986) Rate constants for the reactions of ATP- and ADP-actin with the ends of actin filaments. J Cell Biol 103(6 Pt 2):2747-2754.

49. Lo CJ, Leake MC, Pilizota T, \& Berry RM (2007) Nonequivalence of membrane voltage and ion-gradient as driving forces for the bacterial flagellar motor at low load. Biophys $J$ 93(1):294-302.

50. Béven L, et al. (1998) Membrane permeabilisation and antimycoplasmic activity of the 18-residue peptaibols, trichorzins PA. Biochim Biophys Acta 1372(1):78-90.

51. Griniuviene B, Chmieliauskaite V, \& Grinius L (1974) Energy-linked transport of permeant ions in Escherichia coli cells: evidence for membrane potential generation by protonpump. Biochem Biophys Res Commun 56(1):206-213.

52. Mizutani M \& Miyata M (2019) Behaviors and energy source of Mycoplasma 
53. Uenoyama A \& Miyata M (2005) Gliding ghosts of Mycoplasma mobile. Proc Natl Acad

Sci U S A 102(36):12754-12758.

\section{Figures and Tables}

Figure 1. Structural characters of SpeMreB3 and SpeMreB5. $A$, Negative staining EM image of $10 \mu \mathrm{M}$ SpeMreB3 polymerized in the standard buffer $\left(20 \mathrm{mM}\right.$ Tris- $\mathrm{HCl} \mathrm{pH} 7.5$ at $25^{\circ} \mathrm{C}, 100 \mathrm{mM}$ $\mathrm{KCl}, 5 \mathrm{mM}$ DTT, $2 \mathrm{mM} \mathrm{MgCl}$, $2 \mathrm{mM} \mathrm{ATP}$ ) at $24-27^{\circ} \mathrm{C}$ for 3 hours. Representative SpeMreB3 filaments are indicated by cyan arrows. $B, 2 D$ averaged image of SpeMreB3 filament. The estimated subunit repeat and filament width are $5.3 \pm 0.2$ and $8.7 \pm 0.1 \mathrm{~nm}$, respectively. A weak electron density connecting the protofilaments is indicated as an asterisk. $C-D$, Negative staining EM image of $(C) 5 \mu \mathrm{M}$ and $(D) 10 \mu \mathrm{M}$ SpeMreB5 polymerized in the standard buffer at $24-27^{\circ} \mathrm{C}$ for 3 hours. In panel C, representative raft structures of SpeMreB5 were indicated by red arrows. The boxed region on a paracrystal is magnified as the inset. $E, 2 \mathrm{D}$ averaged image of the SpeMreB5 raft structure. The estimated subunit repeat and the entire width of the raft are $5.3 \pm$ 0.1 and $17.3 \pm 0.1 \mathrm{~nm}$, respectively. A weak electron density connecting the protofilaments corresponding to that of the SpeMreB3 filament is indicated as an asterisk. $F$, Protofilament structures in the crystals of the SpeMreB3 AMPPNP complex and Nf-SpeMreB3. Two different conformations (Mol A and B) in the asymmetric unit of the SpeMreB3 AMPPNP complex crystal are shown in left and center panels. Two subunits in the protofilaments are labeled as $i$ and $i-1$. The subunit repeat is indicated at the right of each $i-1$ subunit. The four subdomains (IA, IB, IIA, and IIB) are labeled on i subunit. $\mathrm{G}-\mathrm{H}$, Close up view of the subunit interface in the protofilament in the crystal of $(G)$ Nf-SpeMreB3 and $(H)$ the SpeMreB3 AMPPNP complex Mol A. Subdomains IA, IB, IIA, and IIB are indicated by ribbon representations colored with pale blue, orange, magenta, and green, respectively. Hydrogen bonds and electrostatic interactions are indicated by broken lines. The residues involved in the hydrogen bonding or electrostatic interaction network are indicated by stick models or blue (main chain nitrogen atom) or red (main chain oxygen atom) spheres with labels. Water molecules involved in the interactions are shown with red spheres. I, Structural comparison of Mol A and B in the SpeMreB3 AMPPNP complex and Nf-SpeMreB3. The structures are superimposed to subdomains IIA and IIB of Mol A. Movement of the subdomain IA and IB is indicated by red arrows. $J$, A ribbon representation of the structure of the antiparallel double-stranded filament of SpeMreB3. K, Electrostatic potential surfaces of the protofilaments of Mol A of the SpeMreB3 AMPPNP complex (top) and the SciMreB5 AMPPNP complex (PDB: 7BVY) (bottom). Three subunits are shown. The four subdomains are labeled on the central subunits in the protofilament.

Figure 2. Polymerization of SpeMreB3 and SpeMreB5 monitored by light scattering. $A-B$, Time course polymerization of $(A)$ SpeMreB3 and $(B)$ SpeMreB5 from 1 to $12 \mu \mathrm{M}$. Right side panels of each graph are enlarged images of boxed regions. Lag phases are indicated by boxes with black solid lines in right side panels. $C$, The long-time polymerization profile of $10 \mu \mathrm{M}$ SpeMreB3 (blue) and SpeMreB5 (red), and $5 \mu \mathrm{M}$ SpeMreB5 (pink). D, Concentration dependence of steady state light scattering of SpeMreB3 (cyan) and SpeMreB5 (red). Error bars indicate S.D. from five repeated measurements. Critical concentrations of each SpeMreB were estimated from the $x$-intercept of each linear fit and estimated as $0.66 \pm 0.28 \mu \mathrm{M}$ for SpeMreB3 and $0.42 \pm 0.05 \mu \mathrm{M}$ for SpeMreB5.

Figure 3. Sedimentation assay of the SpeMreBs. $A-B$, Sedimentation assay over the range of $(A)$ SpeMreB3 and $(B)$ SpeMreB5 concentrations. Each SpeMreB was incubated for 3 hours at $24-27^{\circ} \mathrm{C}$ after initiating polymerization and subjected to the ultracentrifugation of $264,000 \times g, 120$ min, at $23^{\circ} \mathrm{C}$. Pellets were resuspended with a buffer equivalent amount to the samples. Each fraction was quantified by Coomassie stained 12.5\% Laemmli gel. C, Pelleted SpeMreB concentrations were plotted over the total SpeMreB3 (cyan) and SpeMreB5 (red) concentrations with linear fitting. Error bars indicate S.D. from three repeated measurements. Critical 
concentrations were estimated for $0.88 \pm 0.09 \mu \mathrm{M}$ (for SpeMreB3) and $0.18 \pm 0.11 \mu \mathrm{M}$ (for SpeMreB5), as the $x$-intercept of each linear fit. $D$, The bias of SpeMreB3 concentration in the supernatant fraction after the ultracentrifugation of $264,000 \times \mathrm{g}$ for $120 \mathrm{~min}$ at $23^{\circ} \mathrm{C}$. (Left) The scheme of the experiment. The supernatant fraction was divided into upper and bottom halves as described. The whole supernatant fraction (sup mixed) was prepared by 1:1 mixture of the upper and bottom half fractions. (Right) Samples were visualized by Coomassie stained $12.5 \%$ Laemmli gel. Each lane shows the upper half of the supernatant, bottom half of the supernatant, sup mixed, and pellet fractions from left to right. The right most lane $(\mathrm{M})$ is a protein size standard. The SpeMreB3 concentration of upper half, bottom half, and mix of the supernatant were estimated for $0.79 \pm 0.39,8.14 \pm 1.14,4.47 \pm 0.72 \mu \mathrm{M}$, respectively, and the one of pellet was $5.53 \pm 0.72 \mu \mathrm{M}$, from three data sets.

Figure 4. $P_{i}$ release measurements of SpeMreB3 and SpeMreB5 in standard buffer. $A$, Time course plots of $P_{i}$ release of $3 \mu M$ SpeMreB3 wild type (cyan), SpeMreB3 ${ }^{D 147 E}$ (pale blue), SpeMreB3 ${ }^{\mathrm{K} 174 \mathrm{~T}}$ (navy blue), SpeMreB3 ${ }^{\mathrm{K} 174 \mathrm{~T} / \mathrm{S} 176 \mathrm{D}}$ (purple), SpeMreB5 (red), and SpeMreB5 ${ }^{\mathrm{D} 162 \mathrm{~S}}$ (pink) in the presence of $2 \mathrm{mM} \mathrm{ATP} \mathrm{shown} \mathrm{in} \mathrm{solid} \mathrm{lines.} \mathrm{Time} \mathrm{course} \mathrm{polymerization} \mathrm{of} \mathrm{wild} \mathrm{type}$ SpeMreB3 (cyan) and SpeMreB5 (red) are shown in dotted lines. B, Pi release rates of SpeMreBs estimated from panel A. Error bars indicate S.D. from three repeated measurements. Symbols indicate $p$-value supported by Student's $t$-test ${ }^{* *} ; p<0.01$, n.s.; $\left.p>0.05\right)$. $C$, Pi release (red solid line) and polymerization (red dashed line) of $3 \mu \mathrm{M}$ SpeMreB5 in the presence of $3 \mu \mathrm{M}$ ATP. $\mathrm{P}_{\mathrm{i}}$ release of $3 \mu \mathrm{M}$ SpeMreB5 in the presence of $2 \mathrm{mM}$ ATP is shown as the black-colored solid line. $D$, Sedimentation assay of SpeMreB5 over a range of concentrations polymerized in the presence of equal concentrations of ATP. Supernatant and pellet fractions were separated with centrifugation after 3 hours of polymerization, which is long enough to hydrolyze and consume all ATP at each condition (shown with red lines in C). E, Pelleted SpeMreB5 concentrations were plotted over the total SpeMreB5 concentrations with linear fitting. Error bars indicate S.D. from three repeated measurements. Critical concentrations were estimated at $2.31 \pm 0.53 \mu \mathrm{M}$ as the $x$ intercept of each linear fit. $F-H$, Close up view of the nucleotide hydrolysis sites of: $(F)$ the CcMreB AMPPNP complex (PDB: 4CZJ), $(G)$ the SpeMreB3 AMPPNP complex (Mol A), and $(H)$ the SciMreB5 AMPPNP complex (PDB: 7BVY). $\mathrm{Mg}^{2+}$ and water molecules are indicated as green and red spheres, respectively. I, Weblogos of amino acid sequences around the ATP hydrolysis region on (Left) 4832 MreBs from non-Spiroplasma bacteria used in a previous study (9), (Center) 29 Spiroplasma MreB3, and (Right) 171 Spiroplasma MreBs except for MreB3. Corresponding amino acids for the core amino acids for ATP hydrolysis (E140, T167, and E169 in CcMreB) are indicated by triangles.

Figure 5. Working model of SpeMreB polymerization. $A-B$, Scheme of $(A)$ SpeMreB3 and $(B)$ SpeMreB5 polymerization. ATP, ADP, and $\mathrm{P}_{\mathrm{i}}$ are indicated as purple rectangles, navy blue rectangles, and orange circles, respectively. Both SpeMreB3 and SpeMreB5 assemble into filaments, and hydrolyze ATP at filamentous form. SpeMreB3 forms double stranded filaments with short lengths. SpeMreB5 assembles rafts, in which outer SpeMreB5 subunits in the raft structure interact in a different manner to the central interaction. A cycle of polymerization, $\mathrm{Pi}_{\mathrm{i}}$ release and depolymerization of SpeMreB3 and SpeMreB5 are indicated with arrows with solid (monitored in this study) and dotted (putative) lines. The factors to stimulate SpeMreB5 paracrystal formations are $\mathrm{Mg}^{2+}$ and high SpeMreB5 concentrations. 
bioRxiv preprint doi: https://doi.org/10.1101/2021.04.07.438887; this version posted April 9, 2021. The copyright holder for this preprint (which was not certified by peer review) is the author/funder, who has granted bioRxiv a license to display the preprint in perpetuity. It is made

Fig 1
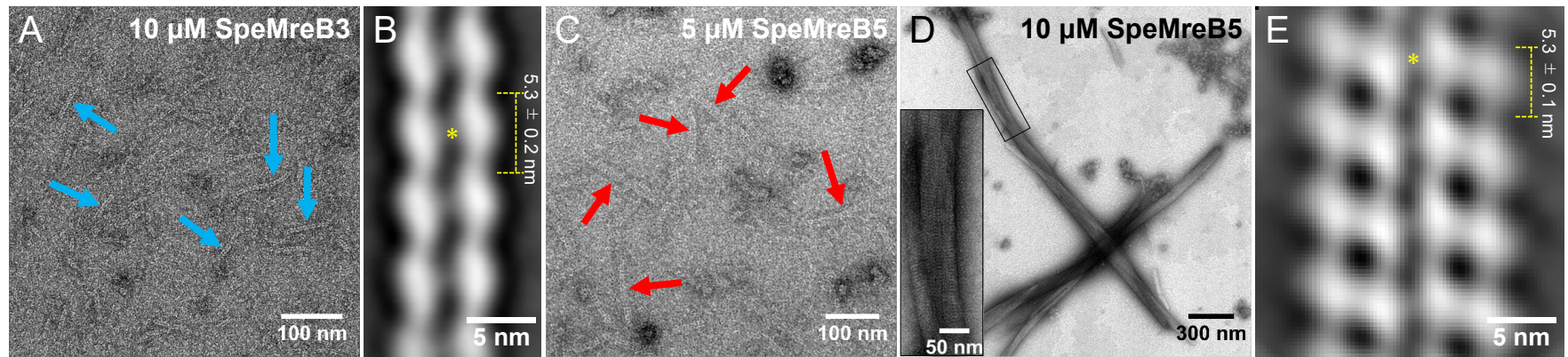

F AMPPNP Mol A

AMPPNP Mol B

Nucleotide free $(\mathrm{Nf})$
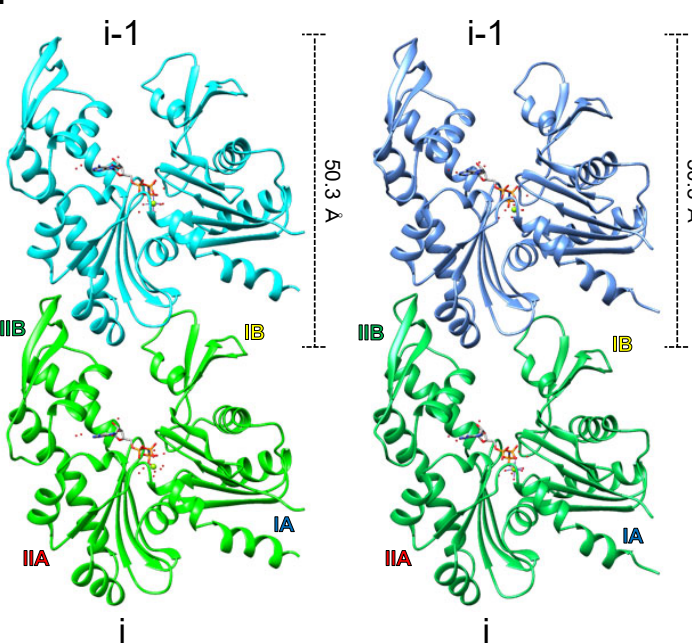

$z^{\mathrm{i}-1}$

G

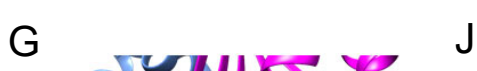

J $\sim$

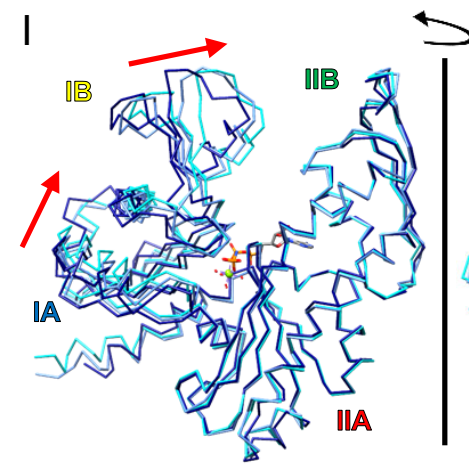

$\prod_{18} 90^{\circ}$
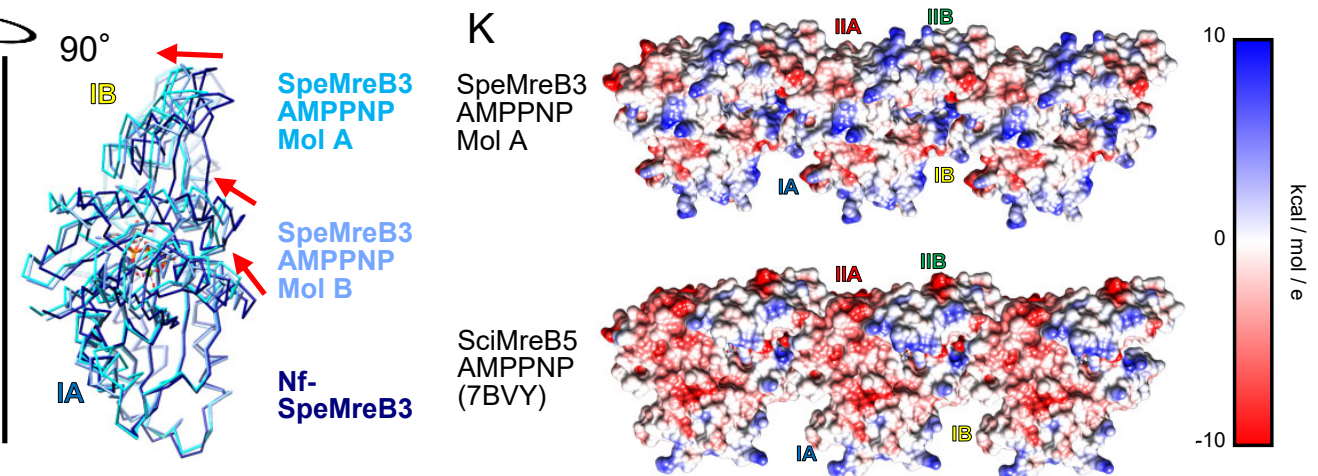
bioRxiv preprint doi: https://doi.org/10.1101/2021.04.07.438887; this version posted April 9, 2021. The copyright holder for this preprint (which Fig 2

A ${ }^{80}$ SpeMreB3
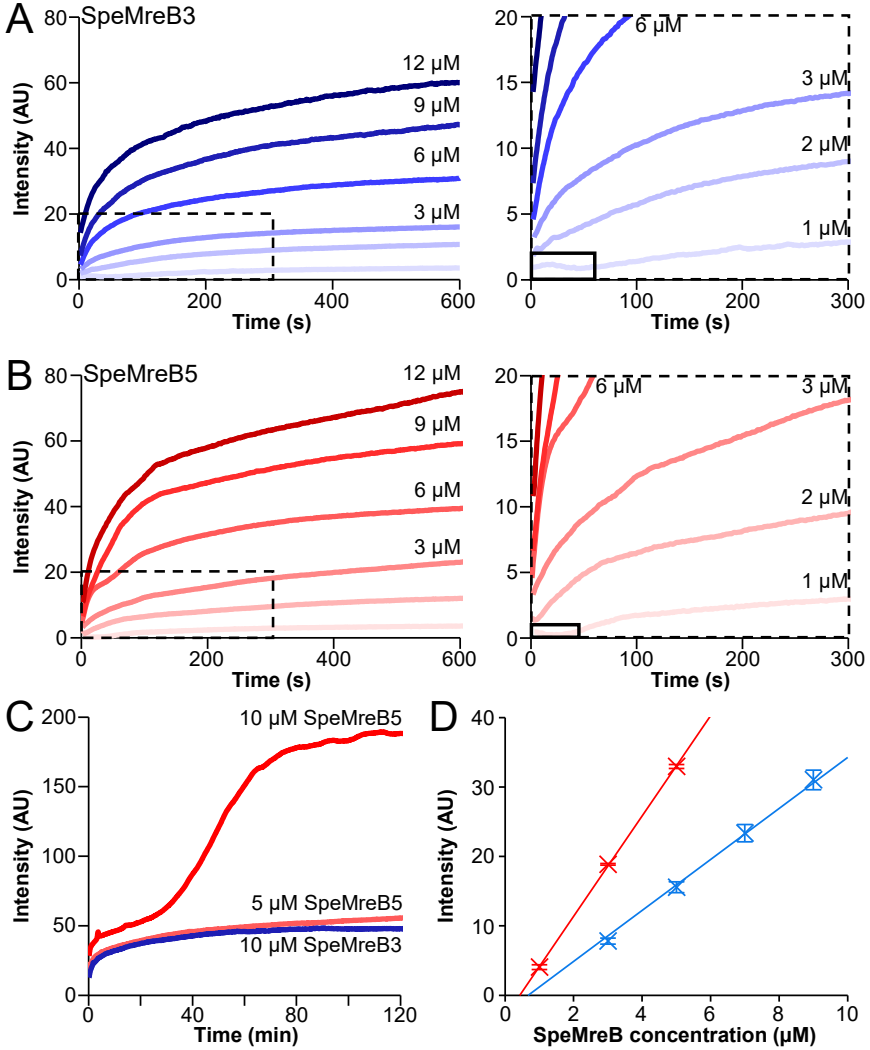
bioRxiv preprint doi: https://doi.org/10.1101/2021.04.07.438887; this version posted April 9, 2021. The copyright holder for this preprint (which was not certified by peer review) is the author/funder, who has granted bioRxiv a license to display the preprint in perpetuity. It is made

Fig 3

A

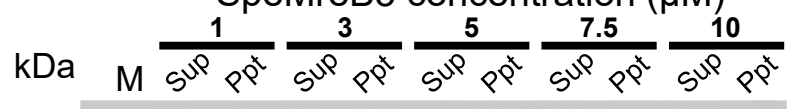

50

37

25

B SpeMreB5 concentration $(\mu \mathrm{M})$

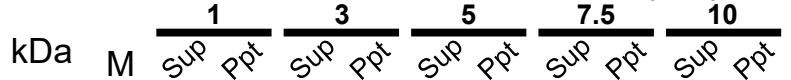

50

37

25

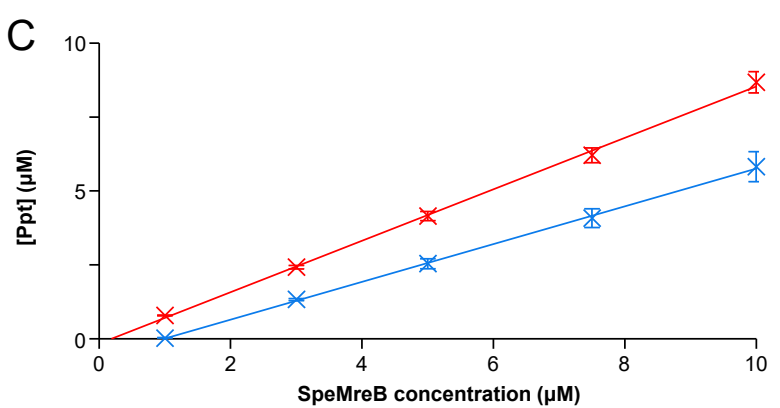

D

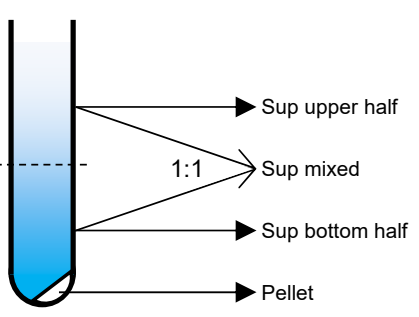

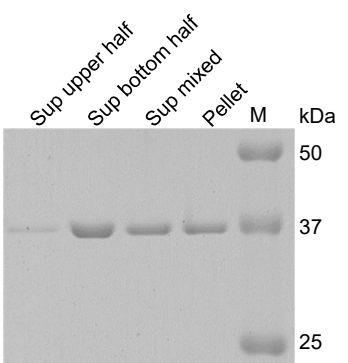


bioRxiv preprint doi: https://doi.org/10.1101/2021.04.07.438887; this version posted April 9, 2021. The copyright holder for this preprint (which Fig 4
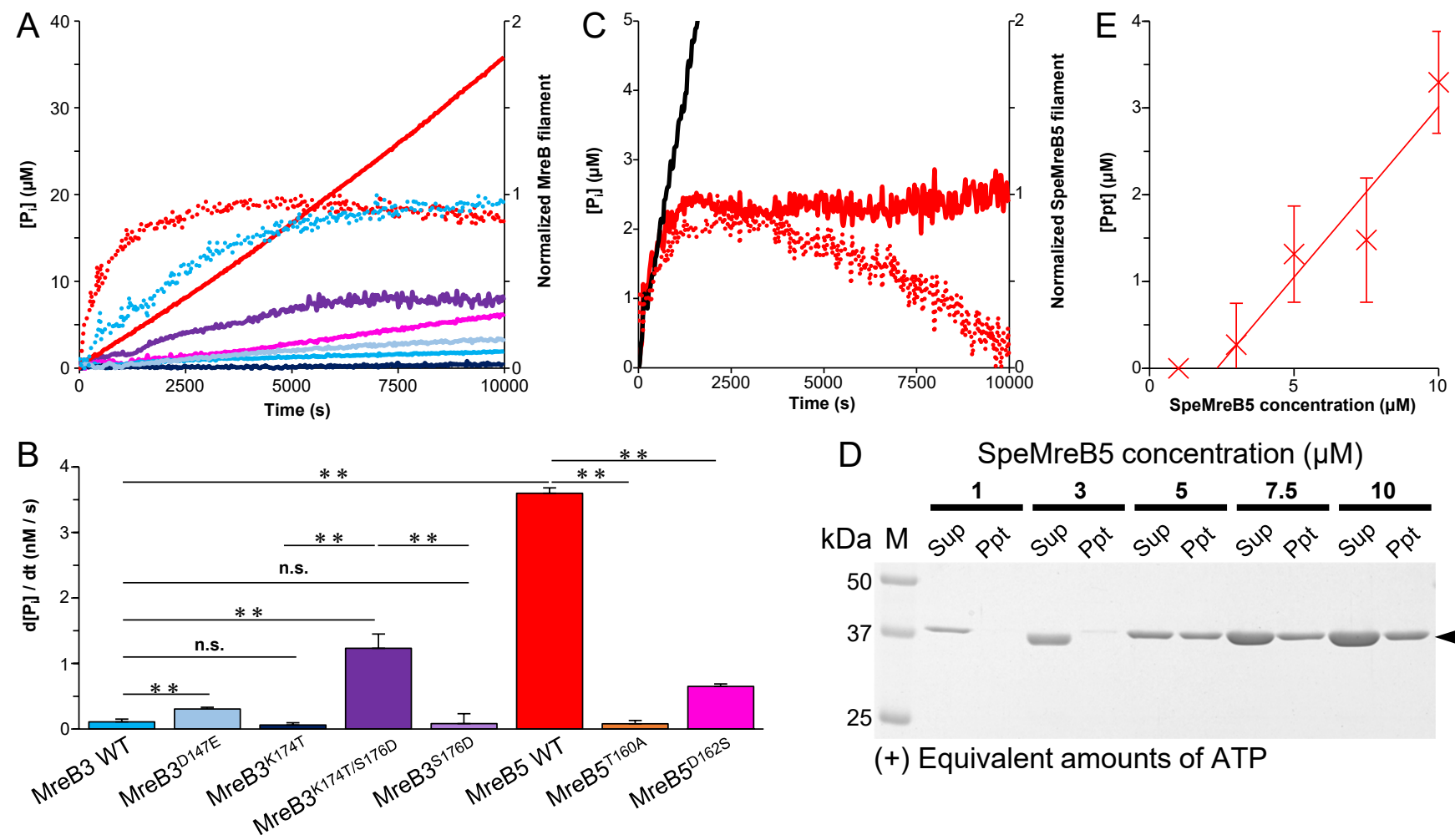

D SpeMreB5 concentration $(\mu \mathrm{M})$

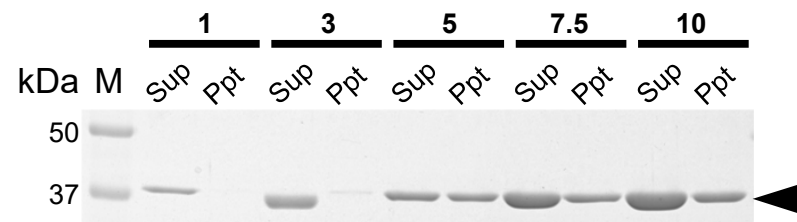

25
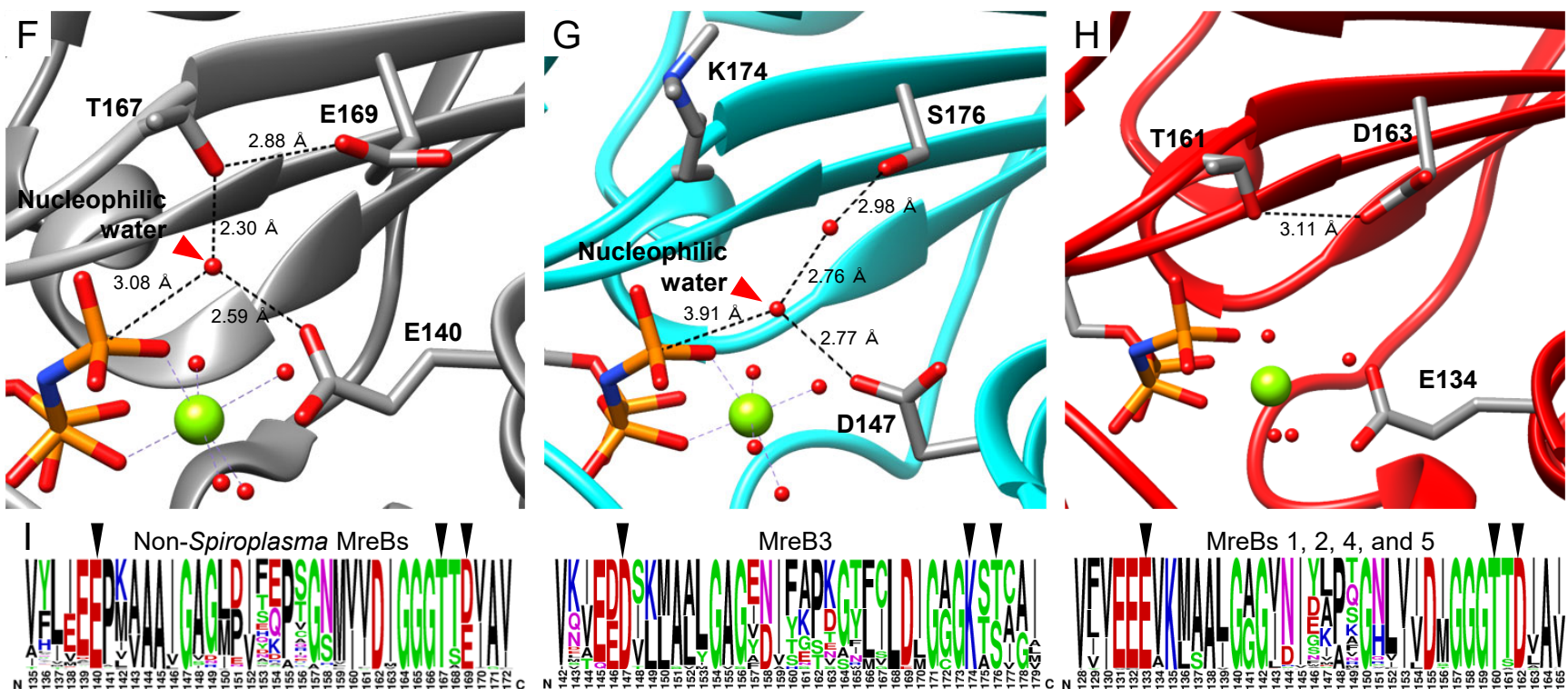

I

MreB3

I

MreBs 1, 2, 4, and 5
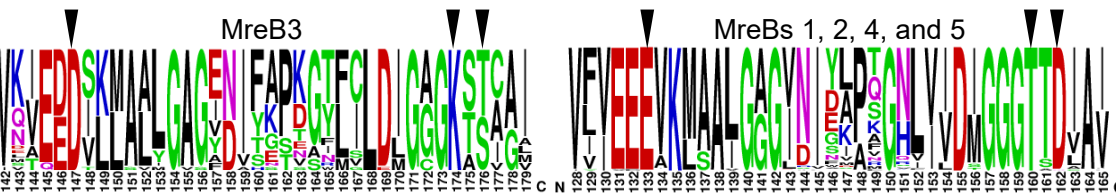
bioRxiv preprint dol: https://doi.org/10.1101/2021.04.07.438887; this version posted April 9, 2021. The copyright holder for this preprint (which was not certified by peer review) is the author/funder, who has granted bioRxiv a license to display the preprint in perpetuity. It is made Fig 5 available under aCC-BY 4.0 International license.

A SpeMreB3

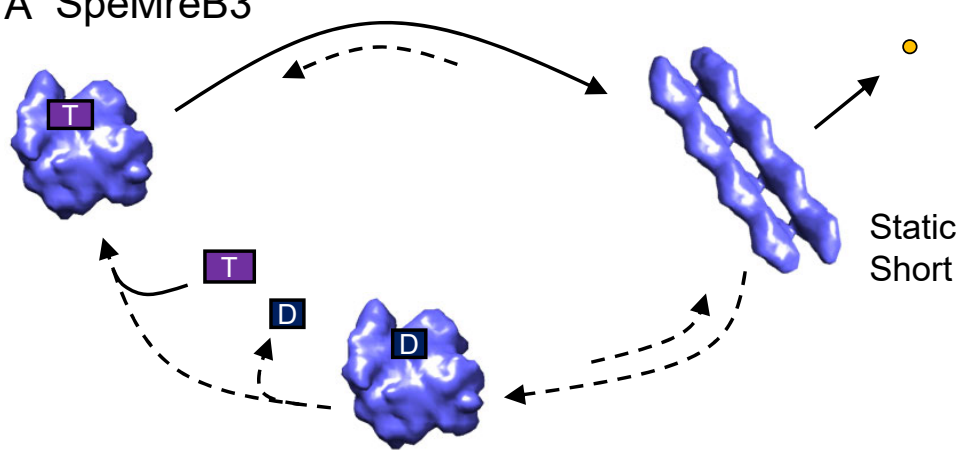

SpeMreB3 T ATP

D ADP

B SpeMreB5
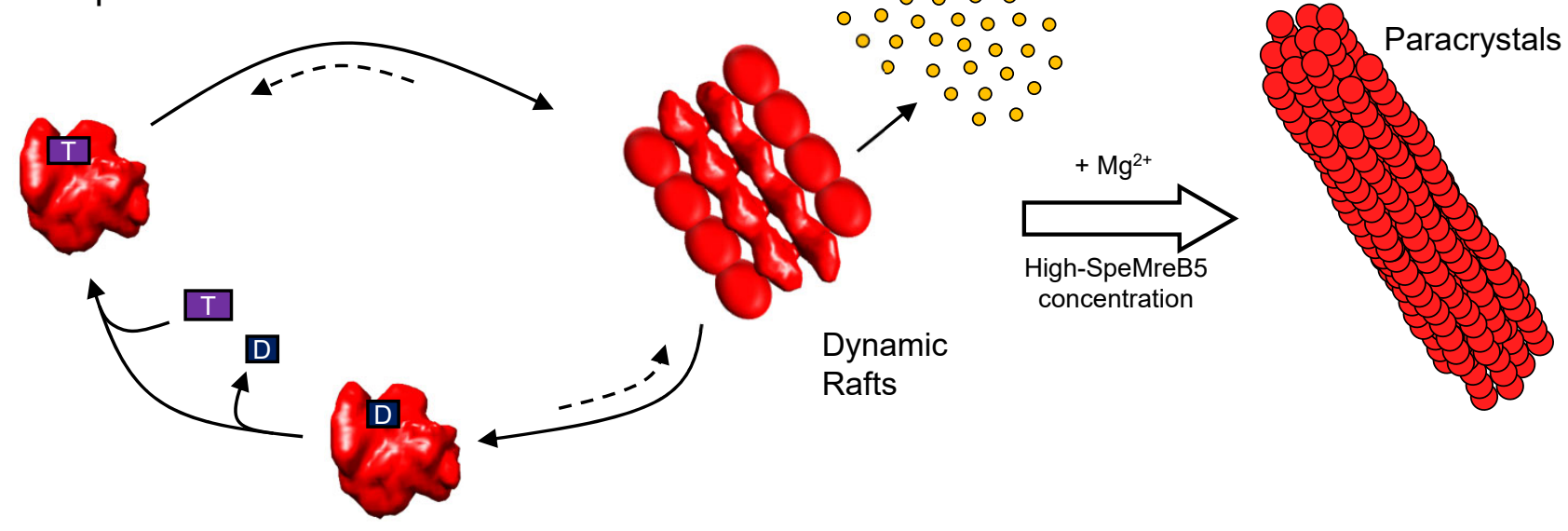